\title{
Neurocognition of Aged Patients With Chronic Tinnitus: Focus on Mild Cognitive Impairment
}

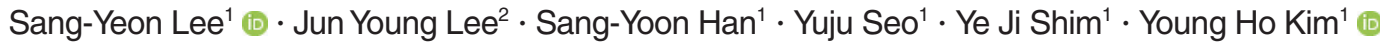 \\ Departments of ${ }^{1}$ Otorhinolaryngology-Head and Neck Surgery and ${ }^{2}$ Psychiatry, SMG-SNU Boramae Medical Center, \\ Seoul National University College of Medicine, Seoul, Korea
}

Objectives. To investigate the neurocognition of aged patients with chronic tinnitus and reveal the possible association between tinnitus severity and cognitive function, with attention to mild cognitive impairment (MCI).

Methods. Fifty-eight elderly patients ( $\geq 65$ years old) with chronic tinnitus ( $\geq 6$ months) were prospectively enrolled in this study. All patients assessed the neurocognitive batteries including the Korean version of the patient health questionnaire-9 (K-PHQ-9), the Lawton instrumental activities of daily living scale (K-IADL), and the Montreal cognitive assessment (MoCA-K). After initial evaluation to exclude moderate or severe cognitive impairment by a psychiatrist, the patients were classified into two groups: $\mathrm{MCI}$ and non-MCI, according to the MoCA-K scores (cutoff value, 22/23). All patients underwent audiological examinations including psychoacoustic tests of tinnitus.

Results. Of 58 patients, $10(17.2 \%)$ met the $\mathrm{MCI}$ criteria. The tinnitus handicap inventory (THI) score in the MCI group was significantly higher than that in the non-MCI group. Based on multivariate regression analysis, a significant association between tinnitus severity and MoCA-K score was also detected. Specifically, bothersome tinnitus (THI score 230) was closely linked to the presence of MCI. Meanwhile, the impact of MCI on both K-PHQ-9 and K-IADL scores was not evident in patients with chronic tinnitus.

Conclusion. Tinnitus severity appears to be a potential independent determinant for predicting the MCI, suggesting the underlying mechanism between chronic tinnitus and cognitive deficit. Given that MCI highly links to dementia, the evaluation of cognitive functions in aged patients with chronic tinnitus need to be considered at the initial assessment of tinnitus.

Keywords. Tinnitus; Mild Cognitive Impairment; Montreal Cognitive Assessment

\section{INTRODUCTION}

Non-pulsatile tinnitus is a common otologic symptom, characterized by conscious auditory perception in the absence of an external stimulus; this is often called phantom sound as there is no corresponding genuine physical source of the sound [1]. Although the exact pathophysiology remains elusive, phantom

\footnotetext{
- Received December 19, 2018

Revised March 12, 2019

Accepted April 4, 2019

- Corresponding author: Young Ho Kim

Department of Otorhinolaryngology-Head and Neck Surgery, SMG-SNU

Boramae Medical Center, 20 Boramae-ro 5-gil, Dongjak-gu, Seoul 07061, Korea

Tel: +82-2-870-2442, Fax: +82-2-870-3863

E-mail: yhkiment@gmail.com
}

sound has been postulated to be caused by maladaptive cortical neural plasticity and resulting hyperactivity that reflects a complex neural network interplay between auditory and non-auditory cortices [2-4]. The constant awareness of this phantom auditory perception often causes considerable distress. In addition to psychological complications, such as social and emotional difficulties, previous studies have also reported cognitive impairment in patients with chronic tinnitus [5-8].

Cognitive function typically involves attention, concentration, use of working memory and information processing [6]. Several studies have evaluated the association between cognitive impairment and chronic tinnitus; tinnitus patients showed poor performance in various cognitive tasks involving attention and working memory [9-11]. A significant correlation between cognitive processing speed and tinnitus handicap inventory (THI)

Copyright $\odot 2020$ by Korean Society of Otorhinolaryngology-Head and Neck Surgery.

This is an open-access article distributed under the terms of the Creative Commons Attribution Non-Commercial License (https://creativecommons.org/licenses/by-nc/4.0)

which permits unrestricted non-commercial use, distribution, and reproduction in any medium, provided the original work is properly cited. 
was found in patients with bothersome tinnitus (THI score $\geq 30$ ) [12]. Recently, electroencephalography (EEG) revealed that neural changes in the hippocampus, anterior cingulate, and insula were associated with cognitive deficits in patients with chronic tinnitus [13]. Hence, neurophysiological changes in patients with tinnitus might be affected by the nature of cognitive deficits.

Despite the existence of putative evidence between tinnitus and cognitive deficits, only a few published studies have yet explored the cognitive function of chronic tinnitus patients [11, $12,14,15]$. Notably, there was no study that suggested the possible association between chronic tinnitus and mild cognitive impairment (MCI). MCI is a clinical syndrome thought to represent the intermediate cognitive state between normal aging and dementia. Patients aged $\geq 65$ years diagnosed with $\mathrm{MCI}$ are more likely to develop dementia than normal elderly people [16]. When patients are diagnosed with MCI, nondisabled level of cognitive decline in their activities of daily living or instrumental activities of daily living are maintained. However, their cognitive abilities tend to deteriorate at a rate in-between that of normal elderly people and mild Alzheimer disease patients, resulting in a higher risk of dementia [17]. Hence, evaluating the presence of MCI in patients with chronic tinnitus may clarify these issues. Thus, we aimed to investigate the cognitive and psychological characteristics of aged patients with chronic tinnitus and to analyze the causal link between tinnitus and MCI.

\section{MATERIALS AND METHODS}

\section{Patients}

From March 2017 to March 2018, consecutive chronic tinnitus ( $\geq 6$ months) patients aged 65 years or more were prospectively enrolled. We excluded cases with (1) hearing loss $>40 \mathrm{~dB}$ in at least one ear (calculated by averaging the pure-tone audiometry [PTA] thresholds at $0.5,1,2$, and $4 \mathrm{kHz}$ ); (2) high-frequency hearing loss (increasing thresholds from 0.25 to $8 \mathrm{kHz}$ and the

\section{H}

- For the first time, we investigated the possible association between tinnitus and cognitive function, with attention to mild cognitive impairment (MCI).

- Of 58 elderly patients with chronic tinnitus, 10 (17.2\%) met the MCI criteria (based on Korean version of the Montreal cognitive assessment [MoCA-K] <23).

- The tinnitus handicap inventory score in the MCI group was significantly higher than that in the non-MCI group.

- A significant negative correlation was found between tinnitus severity and MoCA-K score; given that MCI highly links to dementia, the evaluation of cognitive functions in aged patients with chronic tinnitus need to be considered at the initial assessment of tinnitus. difference between the thresholds at 250 and $8,000 \mathrm{~Hz}$ was $>20$ dB HL) [18]; (3) known history of neuropsychiatric or brain disorder; (4) current (within 3 months) psychotropic or central nervous system-active medications; (5) alcohol or drug abuse; (6) hearing aids or sound generators that may influence hearing and tinnitus level.

Finally, 58 eligible patients were enrolled in this study. All patients were assessed using the MoCA-K, a Korean version of the Montreal cognitive assessment designed to screen for MCI. After further excluding the moderate or severe cognitive impairment by a psychiatrist (JYL), patients were classified into the MCI group ( $\mathrm{n}=10$; MoCA-K score $<23$ ) or into the non-MCI group $(n=48$; MoCA-K score $\geq 23$ ) based on a cutoff value of 22 [19]. This study was approved by the Institutional Review Board of the SMG-SNU Boramae Medical Center (IRB No. 16-2016-401), and patient consent was waived.

\section{Audiological and psychoacoustic evaluations}

At the initial visit, a structured history of the characteristics of tinnitus on the affected side and the psychoacoustic nature (pure-tone or narrow-band noise) of tinnitus was obtained. All subjects underwent PTA testing that included psychoacoustic tests of tinnitus, such as tinnitus pitch and loudness matching. The hearing thresholds for seven different octave frequencies $(0.25,0.5,1,2,3,4$, and $8 \mathrm{kHz})$ were evaluated using PTA in a soundproof booth. The mean hearing threshold was calculated using the average of the hearing thresholds at $0.5,1,2$, and 4 $\mathrm{kHz}$. For analysis, we selected the hearing threshold in the tinnitus affected side. In cases of patients with bilateral tinnitus, we documented the mean hearing threshold in the dominant tinnitus side. The perceived tinnitus handicap was measured using THI. Based on a previous study, bothersome tinnitus was defined as THI $\geq 30$ [12]. We also assessed the visual analog scale (VAS) scores (patients answering questions on four aspects of tinnitus such as loudness, annoyance, awareness, and effect of life during daytime on a scale from 0 to 10 ).

\section{Neurocognitive evaluation}

All patients underwent a battery of neurocognitive tests, including three questionnaires at initial assessment for tinnitus: Korean version of the patient health questionnaire-9 (K-PHQ-9), Korean version of the Lawton instrumental activities of daily living scale (K-IADL), and MoCA-K.

\section{MoCA-K}

MoCA-K is a Korean version of rapid screening neurocognitive test for MCI. It can be completed in 10 minutes. It scores from 0 to 30 , with high scores indicating better cognition. Based on a previous study employing neuropsychological batteries for MCI screening, the MoCA-K displayed excellent sensitivity (89\%) and good specificity $(84 \%)$ with the implementation of the cutoff value of 22 [19]. Thus, we decided to define $\mathrm{MCI}$ as an MoCA-K 
score below 23. Attention and concentration, executive functions, memory, language, visuoconstructional skills, conceptual thinking, calculation, and orientation were assessed. In the MoCA-K, the words used in the short-term memory recall task and the letters used in the trail-making B task were changed, and the phonemic fluency task was replaced with a semantic fluency task. One point was added for participants with 6 or fewer years of education instead of 12 or fewer years of education. The other tests were similar to those in the original MoCA [19].

\section{K-PHQ-9}

PHQ was developed for the prompt diagnosis of neuropsychiatric disorders [20]. PHQ-9, a simple self-reporting assessment for depression screening, primarily focuses on depression severity measurement. The score ranges from 0 to 27 , and the completion time is less than 3 minutes. PHQ-9 comprises of nine items and is used for depression diagnosis according to the diagnostic and statistical manual of mental disorders, fifth edition (DSM-IV). In this study, all patients underwent assessment using the K-PHQ-9. According to previous studies, the optimal cutoff score for major depression is $<10$ in PHQ-9, with $88.5 \%$ sensitivity and 94.7\% specificity [21]. Moreover, K-PHQ-9 has been reported as a reliable and effective tool for screening depression [22].

\section{K-IADL}

The K-IADL, a Korean version of a self-reporting questionnaire to assess difficulties in performing instrumental activities of daily living, was given to all patients. K-IADL comprises of 11 items, with the score of each item ranging from 0 to 3 as follows: 0 , normal; 1 , with some assistance; 2 , with much assistance; 3, unable to do; NA, not applicable. The K-IADL consists of eleven self-evaluating questions concerning capacity and difficulty in performing activities of daily living, including (1) shopping, (2) mode of transportation, (3) ability to handle finances, (4) housekeeping, (5) preparing food, (6) ability to use a telephone, (7) responsibility for own medication, (8) recent memory, (9) hobbies, (10) watching television, and (11) fixing things around the house. Based on a previous study, K-IADL showed excellent internal consistency and test-to-test reliability. K-IADL has been reported as a valid and reliable tool for evaluating functional status in the elderly, especially when using a cutoff of 10 in measuring daily living activities [23]. Moreover, K-IADL findings have been consistent with other general cognitive indices, demonstrating good validity [23].

\section{Statistical analysis}

All data were analyzed using IBM SPSS ver. 22.0 (IBM Corp., Armonk, NY, USA). To determine significant differences between the two groups in the screened continuous and categorical variables, independent Student $t$-tests, Mann-Whitney $U$-test and chi-square tests were performed, as appropriate. To analyze the association between tinnitus severity (based on THI score)
Table 1. Demographics and clinical feature of the subjects

\begin{tabular}{lccc}
\hline Variable & $\begin{array}{c}\mathrm{MCl} \text { group } \\
(\mathrm{n}=10)\end{array}$ & $\begin{array}{c}\text { Non-MCl group } \\
(\mathrm{n}=48)\end{array}$ & $P$-value \\
\hline Sex & & & 0.287 \\
$\quad$ Male & 2 & 21 & \\
$\quad$ Female & 8 & 27 & \\
Age (yr) & $70.9 \pm 7.9$ & $\begin{array}{c}67.5 \pm 4.1 \\
(65-82)\end{array}$ & 0.211 \\
& $(65-84)$ & \\
Tinnitus duration (yr) & $2.0(0.5-15)$ & $2.0(0.5-20)$ & 0.731 \\
Audiologic evaluation & & & \\
$\quad$ Hearing threshold $(\mathrm{dB} \mathrm{HL})$ & $33.0 \pm 7.4$ & $25.7 \pm 8.7$ & 0.017 \\
$\quad$ Speech discrimination (\%) & $91.6 \pm 12.4$ & $93.3 \pm 7.2$ & 0.407 \\
\hline
\end{tabular}

Values are presented as mean $\pm \mathrm{SD}$ (range), median (range), or mean $\pm \mathrm{SD}$. $\mathrm{MCl}$, mild cognitive impairment; $\mathrm{HL}$, hearing loss; SD, standard deviation.

and cognitive deficits (based on MoCA-K score), Pearson correlation analysis was performed. Multivariate and logistic regression analysis was performed to simultaneously assess the relative influence of cognitive deficit and associated variables including the age, sex, duration of tinnitus, and mean hearing threshold. $P$-values $<0.05$ were considered to indicate statistical significance.

\section{RESULTS}

\section{Demographic and clinical characteristics}

The patients' demographic and clinical characteristics are presented in Table 1. The mean age of the patients was $68.1 \pm 5.1$ years (range, 65 to 82 years). The number of male and female was 23 $(39.7 \%)$ and $35(60.3 \%)$, respectively. The median duration of tinnitus was 2 years (range, 0.5 to 20 years). The rate of MCI based on MoCA-K was 10 out of 58 (17.2\%). When comparing between the $\mathrm{MCI}$ and non-MCI groups, no significant difference in age (70.9 \pm 7.9 vs. $67.5 \pm 4.1$ years, $P=0.211)$, duration of tinnitus $(P=0.731)$, or sex $(P=0.287)$ was detected. There was a significant difference in hearing threshold $(33.0 \pm 7.4$ vs. $25.7 \pm$ $8.7 \mathrm{~dB}, P=0.017$ ) between two groups. But, the speech discrimination $(91.6 \% \pm 12.4 \%$ vs. $93.3 \% \pm 7.2 \%, P=0.407)$ in the $\mathrm{MCI}$ group was similar to that in the non-MCI group. An individualized profile of each subject was presented in Supplementary Table 1.

\section{Comparison of neurocognitive results}

The mean MoCA-K, median K-PHQ-9, and median K-IADL scores from all patients were $24.5 \pm 4.0$ (range, 8 to 29), 3 (range, 0 to 26), and 8 (range, 5 to 14), respectively. The MoCA-K score, as expected, was significantly different $(18.9 \pm 3.2$ vs. $26.1 \pm 1.8$, $P<0.001)$ between the MCI and non-MCI group. On the other hand, the K-IADL and K-PHQ-9 scores showed no significant change between the MCI group and non-MCI group $(P=0.411$ and $P=0.261$, respectively) (Fig. 1). 


\section{Characteristics with respect to tinnitus}

The psychoacoustic characteristics (tinnitus pitch and loudness) of patients between the $\mathrm{MCI}$ group and the non-MCI group were similar (Table 2). Of the 58 patients, 21 (36.2\%) were bothered by their tinnitus according to our set criteria (THI $\geq 30$ ). Notably, the THI score of the MCI group was significantly higher than that of the non-MCI group (33.6 \pm 10.4 vs. $21.9 \pm 10.1, P=0.002)$.

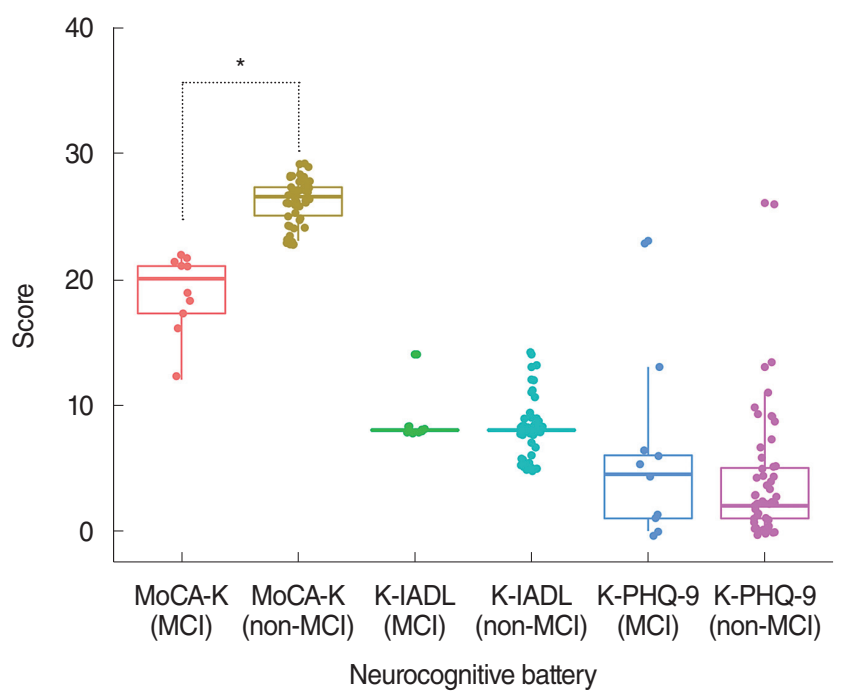

Fig. 1. Comparison of neurocognitive test results based on the presence of mild cognitive impairment ( $\mathrm{MCl}$ ) in aged patients ( $\geq 65$ years) with chronic tinnitus. In the $\mathrm{MCl}$ group, the Korean version of the Montreal cognitive assessment (MoCA-K) score was significantly lower than that in the non-MCI group $(P<0.001)$. However, Korean version of the Lawton instrumental activities of daily living scale (KIADL) and Korean version of the patient health questionnaire-9 (KPHQ-9) scores were similar regardless of the presence of $\mathrm{MCl}(P=$ 0.411 and $P=0.261$, respectively). ${ }^{*} P<0.05$ by independent $t$-test.
Moreover, there was a significant association between MCI (MoCA-K $<23$ ) and bothersome tinnitus (THI $\geq 30$ ) but not in the patients who were not bothered by tinnitus (THI $<30$ ) (Table 3 ). VAS scores, including awareness ( $6.6 \pm 3.2$ vs. $5.8 \pm 3.1, P=0.372)$,

Table 2. Baseline psychoacoustic characteristics and severity of tinnitus

\begin{tabular}{lccc}
\hline Variable & $\begin{array}{c}\mathrm{MCl} \text { group } \\
(\mathrm{n}=10)\end{array}$ & $\begin{array}{c}\text { Non-MCl group } \\
(\mathrm{n}=48)\end{array}$ & $P$-value \\
\hline $\begin{array}{l}\text { Tinnitus laterality } \\
\quad \text { Right }\end{array}$ & $1(10.0)$ & $10(20.8)$ & 0.565 \\
$\quad$ Left & $3(30.0)$ & $14(29.2)$ & \\
Bilateral & $6(60.0)$ & $24(50.0)$ & - \\
Tinnitus pitch (kHz) & & & 0.184 \\
$\quad$ Median (range) & $6(0.125-8)$ & $6(0.125-8)$ & \\
$\quad$ Mean \pm SD & $4.1 \pm 3.6$ & $3.3 \pm 3.5$ & \\
Tinnitus loudness (dB) & $59.9 \pm 19.3$ & $46.6 \pm 19.1$ & 0.064 \\
THI score & $33.6 \pm 10.4$ & $21.9 \pm 10.1$ & $0.002^{*}$ \\
\hline
\end{tabular}

Values are presented as number (\%) or mean \pm SD unless otherwise indicated.

$\mathrm{MCl}$, mild cognitive impairment; SD, standard deviation; THI, tinnitus handicap inventory.

${ }^{\star} P<0.05$ by independent $t$-test.

Table 3. Association between the development of $\mathrm{MCl}$ and bothersome tinnitus in aged patients ( $\geq 65$ years) with chronic tinnitus

\begin{tabular}{lccccc}
\hline & \multicolumn{3}{c}{ Tinnitus } & & \\
\cline { 2 - 5 } & $\begin{array}{c}\text { Bothersome } \\
(\mathrm{THI} \geq 30)\end{array}$ & $\begin{array}{c}\text { Non-bothersome } \\
(\mathrm{THI}<30)\end{array}$ & Total & $\chi^{2}$ & $P$-value \\
\hline+ & 5 & 5 & 10 & 7.384 & $0.03^{*}$ \\
- & 5 & 43 & 48 & & \\
Total & 33 & 39 & 72 & & \\
\hline
\end{tabular}

$\mathrm{MCl}$, mild cognitive impairment; $\mathrm{THI}$, tinnitus handicap inventory. ${ }^{*} P<0.05$ by Fisher's exact test.

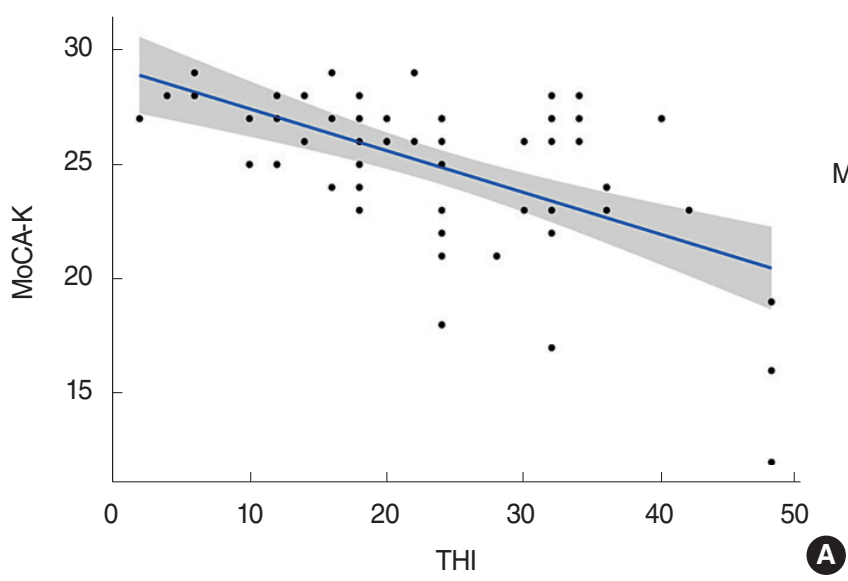

Plot for odds ratio of the final model

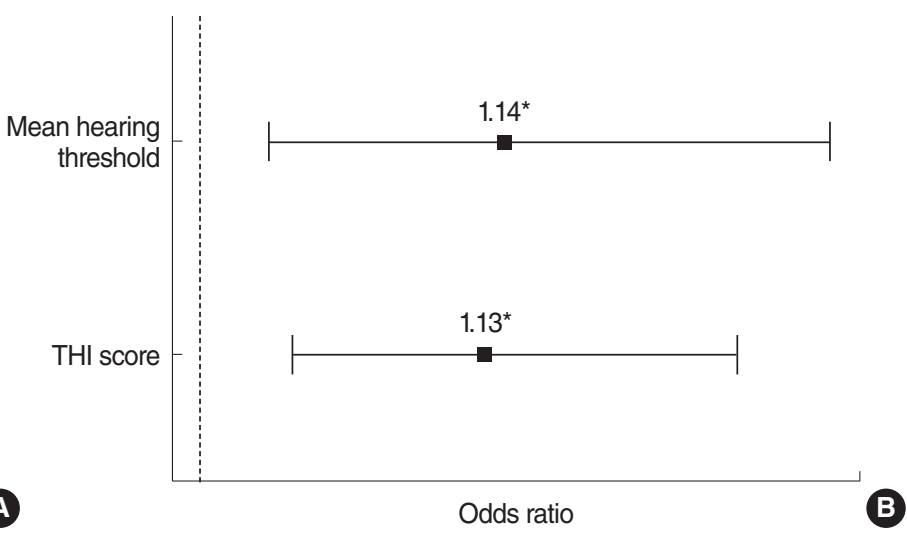

Fig. 2. Association between tinnitus severity and cognitive function in aged patients ( $\geq 65$ years) with chronic tinnitus. (A) A statistically significant negative correlation was detected between tinnitus handicap inventory (THI) and Korean version of the Montreal cognitive assessment (MoCA-K) scores in all patients $(r=-0.837, P<0.001)$. (B) A plot for odds ratios of the final model. As a result of logistic regression analysis, either mean hearing threshold or THI scores significantly contributed to the developing mild cognitive impairment. ${ }^{*} P<0.05$ by logistic regression analysis. 
annoyance $(5.4 \pm 2.9$ vs. $4.0 \pm 2.9, P=0.109)$, loudness $(5.5 \pm 2.6$ vs. $4.4 \pm 2.6, P=0.177)$, and effect of life ( $3.2 \pm 2.4$ vs. $3.0 \pm 2.9$, $P=0.77)$, were not statistically different between the MCI and non-MCI groups.

\section{Association of tinnitus severity and cognitive function}

Pearson coefficient analysis among 58 chronic tinnitus patients revealed a significant negative correlation between THI and MoCA-K scores $(r=-0.837, P<0.001)$ (Fig. 2A). Based on multivariate regression analysis, an increase of THI was significantly associated with a decrease of MoCA-K score (estimate: -0.170 ; standard error, 0.032; $P<0.001$ ), after adjusting age, sex, duration of tinnitus, and mean hearing threshold. In line with this, based on logistic regression analysis, an increase of THI score was significantly associated with the presence of MCI (odds ratio [OR], 1.12; 95\% CI, 1.02 to 1.23; $P=0.014$ ), after adjusting age, sex, duration of tinnitus, and mean hearing threshold. The significant predictors that contribute to the development of MCI were both mean hearing threshold (OR, 1.14) and tinnitus severity (OR, 1.13) (Fig. 2B).

\section{DISCUSSION}

The present study aimed to investigate the association between tinnitus severity and cognitive function, especially in patients with MCI, among aged patients with chronic tinnitus. Ten of 58 patients with chronic tinnitus $(17.2 \%)$ were diagnosed with MCI based on the MoCA-K score (cutoff value, 22). The MCI incidence in this study is similar to that reported by a previous study (10\%-20\% among elderly patients aged $>65$ years) [24]. Based on logistic regression analysis, tinnitus severity is independently associated with the presence of MCI in aged patients with chronic tinnitus. More specifically, a significant association between the presence of MCI and bothersome tinnitus (THI $\geq 30$ ) was found in the present study.

\section{The impact of tinnitus severity on $\mathrm{MCl}$ presence}

Several previous studies have indicated the presence of cognitive impairments, including attention deficit, instant memory disorder, and inefficient learning in patients with chronic tinnitus $[6,15]$. Despite inconsistent results on the association of tinnitus with certain types of cognitive functions, tinnitus has been shown to have negative effects on executive or selective attention and working memory [6]. A study suggested that the presence of chronic tinnitus may be associated with reduced cognitive functioning, particularly attention [25]. The cognitive deficit underlying chronic tinnitus may involve a failure in directing attention away from a phantom sound. Recently, a significant impairment in cognitive control and inhibition was detected in chronic tinnitus patients [13]. In this regard, it is yet to be validated whether chronic tinnitus may increase the risk of MCI by leading to neurocognitive network changes influencing attention-switching. Meanwhile, working memory has a limited capacity that are relevant theoretical composition for cognition [6]. Tinnitus could affect cognition such that it reduces the cognitive capacity available for conducting other tasks [11]. Additionally, both tinnitus and hearing could each separately elicit overload on the central executive [6], which may result in ineffective working memory. From this perspective, even though most patients involved in this study had normal hearing or mild hearing impairments, tinnitus may constitute in the development of MCI, especially in patients with severe tinnitus.

There is a growing body of evidence indicating that the severity of chronic tinnitus is associated with a broad range of cognitive performance. As shown previously, the more severe the tinnitus, the more likely the deterioration of cognitive processing is, suggesting that cognitive processing speed is an independent predictor of tinnitus severity [12]. Furthermore, a neuropsychological study reported a significant negative correlation between tinnitus loudness and duration, and performance on neurocognitive tasks [15]. In this regard, the increase in tinnitus severity may negatively affect attentional orientation and executive control, reducing cognitive processing speed [6]. Similarly, one study reported that patients with severe tinnitus presented with lower scores during cognitive ability screening assessments in almost all subdomains, as compared to mild or moderate tinnitus patients [14]. Moreover, tinnitus severity was negatively correlated with cognition and positively correlated with N2 and P3 latencies of the P300 event-related potential (ERP) [14]. Given that ERPs provide an objective evaluation of attention domains, an increase in tinnitus severity might adversely impact cognitive performance via auditory inattention, which may in turn potentiate cognitive deficits. Consistent with previous studies, the present study demonstrated an association between bothersome tinnitus (THI $\geq 30$ ) and MCI development. Even though the presence of MCI did not negatively affect patients' quality of life in this study, without intervention, severe tinnitus may be associated with serious cognitive deficits, such as dementia. Such deficits may in turn lead to a direct decrease in quality of life and work efficiency. However, this hypothesis awaits further confirmation.

The present study revealed that cognitive impairment is common in tinnitus patients. However, the exact pathophysiology behind the development of tinnitus-associated cognitive deficits has not been yet elucidated. A neuropsychological study investigating cognitive processing in chronic tinnitus patients revealed that tinnitus disrupts non-auditory processing of verbal, visual, and visual-spatial information, suggesting the development of neuroplastic changes [15]. Furthermore, a neuroimaging study employing EEG with source analysis recently reported the nature of the cognitive deficits in tinnitus patients [13]. Notably, a negative correlation was found between neural activity in the hippocampus, the anterior cingulate, and insula, and MoCA 
score [13]. A previous study also reported a negative correlation between changes in EEG activity in cognition-related regions and THI score after tinnitus treatment using a neurophysiological model [3]. The increased neural activity during the resting state in chronic tinnitus patients was correlated with the need for more effort in cognitive processing task completion [13]. Thereby, patients with severe tinnitus are highly likely to present with maladaptive cortical plasticity that may attenuate cognitive performance.

\section{Implication of $\mathrm{MCl}$ screening in aged patients with chronic tinnitus}

The evaluation of cognitive screening tests for patients with severe tinnitus may be beneficial for the appropriate management of both tinnitus and MCI. The impact of tinnitus on quality of life is better defined by identifying the associated cognitive impairments for optimal rehabilitation. Given the high risk of dementia in patients with MCI [26,27], cognitive function testing would allow clinicians to validate hindered cognitive difficulties that may progress to dementia, as well as track any changes in perceived cognitive difficulties and tinnitus distress over the course of treatment. In this study, patients with MCI appeared to experience more difficulties in daily life due to chronic tinnitus compared to patients with normal cognitive function. Taken together, chronic tinnitus may be a potential predictor of MCI that progresses to dementia; thereby, the evaluation of cognitive functions in aged patients with chronic tinnitus need to be considered at the initial assessment of tinnitus.

\section{Limitations and future perspectives}

To the best of our knowledge, this is the first study to examine the cognitive and psychologic aspects of aged patients with chronic tinnitus with a special focus on the presence of $\mathrm{MCI}$, and its association with severe tinnitus. Although we demonstrated significant findings, there are several potential limitations that should be controlled in further investigations.

First, we addressed a correlation between tinnitus severity and MCI; however, we were unable to identify the causal link between the two because we did not evaluate the changes of cognitive function after tinnitus treatment. Further investigation of cognition change according to tinnitus improvement may be necessary to validate this causal association. Additionally, previous study showed that the existence of depression and/or anxiety negatively commonly underlie in tinnitus patients than in healthy controls [28]. In other word, tinnitus-related emotional state disorder has been closely linked to cognitive deficits [29]. Although we evaluated depression status and the activities of daily living using self-reporting questionnaires, variable emotional states might introduce bias and hinder the cognitive ability assessment due to the nature of cross-sectional study. Likewise, we assessed for MCI once during the initial screening. Considering that the cognition of approximately $15 \%-20 \%$ of
MCI patients spontaneously improves without treatments after 1-2 years [26], longer follow-ups are needed to clearly demonstrate the association between tinnitus and MCI. Taken together, it is evident that a comprehensive neuropsychological investigation for chronic tinnitus towards highly complex neurobiological process is necessary.

Second, Lin et al. [30] demonstrated the hazard ratio $(95 \%$ confidence interval) for incident all-cause dementia was 1.89 (1.00 to 3.58 ) for mild hearing loss, compared to normal hearing. Nonetheless, we included the patients with mild hearing impairment due to a severe loss of cohort when performing strict audiological criteria. Although the association of mild hearing loss and MCI remains unknown, our results might be biased by the confounder. Therefore, strict normal hearing criteria may be needed to prove what the authors claim. In a recent study, subjects with tinnitus and severe acquired hearing loss exhibited higher cortical activity in the parahippocampus than did non-tinnitus controls [31]. This increase may be associated with abnormally enhanced attention and impaired memory leading to cognitive deficits. Thus, absence of severe or profound hearing loss in our subjects might hinder the precise result interpretation. Therefore, future studies exploring the role of the degree of hearing in tinnitus-associated MCI should be considered.

Third, the present study did not adopt an objective approach such as neuroimaging or electrophysiology in the examination of the nature of these cognitive deficits. Neurobiological modelling studies have reported that brain areas like the limbic system, autonomic nervous system, and sensory cortex may contribute to the association between tinnitus and cognitive deficits $[15,32]$. Therefore, neurobiological and neuroimaging approaches should be implemented to aid with the identification of the cognitive impairment etiology in chronic tinnitus patients. At present, we are conducting the neurobiological study combined with neuroimaging to reveal the casual link between tinnitus and MCI.

Our results suggest that tinnitus severity was closely linked to cognitive deterioration that results in MCI. Therefore, evaluation of cognitive function in aged patients with chronic tinnitus needs to be considered at the initial assessment to allow for appropriate tinnitus and MCI management.

\section{CONFLICT OF INTEREST}

No potential conflict of interest relevant to this article was reported.

\section{ACKNOWLEDGMENTS}

This study was supported by a clinical research grant provided by SMG-SNU Boramae Medical Center, Seoul, Republic of Korea. 


\section{ORCID}

Sang-Yeon Lee https://orcid.org/0000-0003-3566-8708

Young Ho Kim https://orcid.org/0000-0001-8889-3285

\section{AUTHOR CONTRIBUTIONS}

Conceptualization: YHK. Data curation: YJS, SYH. Formal analysis: SYL. Methodology: SYL, JYL, YHK. Project administration: JYL. Visualization: SYL, YJS, SYH. Writing - original draft: SYL. Writing - review \& editing: JYL, YHK.

\section{SUPPLEMENTARY MATERIALS}

Supplementary materials can be found via https://doi.org/10. 21053/ceo.2018.01914.

\section{REFERENCES}

1. Jastreboff PJ. Phantom auditory perception (tinnitus): mechanisms of generation and perception. Neurosci Res. 1990 Aug;8(4):221-54.

2. Lee SY, Nam DW, Koo JW, De Ridder D, Vanneste S, Song JJ. No auditory experience, no tinnitus: lessons from subjects with congenital- and acquired single-sided deafness. Hear Res. 2017 Oct;354:915.

3. Kim SH, Jang JH, Lee SY, Han JJ, Koo JW, Vanneste S, et al. Neural substrates predicting short-term improvement of tinnitus loudness and distress after modified tinnitus retraining therapy. Sci Rep. 2016 Jul;6:29140.

4. Song JJ, Vanneste S, De Ridder D. Dysfunctional noise cancelling of the rostral anterior cingulate cortex in tinnitus patients. PLoS One. 2015 Apr;10(4):e0123538.

5. Andersson G, McKenna L. The role of cognition in tinnitus. Acta Otolaryngol Suppl. 2006 Dec;(556):39-43.

6. Mohamad N, Hoare DJ, Hall DA. The consequences of tinnitus and tinnitus severity on cognition: a review of the behavioural evidence. Hear Res. 2016 Feb;332:199-209.

7. Pace E, Zhang J. Noise-induced tinnitus using individualized gap detection analysis and its relationship with hyperacusis, anxiety, and spatial cognition. PLoS One. 2013 Sep;8(9):e75011.

8. Tegg-Quinn S, Bennett RJ, Eikelboom RH, Baguley DM. The impact of tinnitus upon cognition in adults: a systematic review. Int J Audiol. 2016 Oct;:55(10):533-40.

9. Trevis KJ, McLachlan NM, Wilson SJ. Cognitive mechanisms in chronic tinnitus: psychological markers of a failure to switch attention. Front Psychol. 2016 Aug;7:1262.

10. Zarenoe R, Hallgren M, Andersson G, Ledin T. Working memory, sleep, and hearing problems in patients with tinnitus and hearing loss fitted with hearing aids. J Am Acad Audiol. 2017 Feb;28(2): 141-51.

11. Rossiter S, Stevens C, Walker G. Tinnitus and its effect on working memory and attention. J Speech Lang Hear Res. 2006 Feb;49(1):
$150-60$.

12. Das SK, Wineland A, Kallogjeri D, Piccirillo JF. Cognitive speed as an objective measure of tinnitus. Laryngoscope. 2012 Nov;122(11): 2533-8.

13. Vanneste S, Faber M, Langguth B, De Ridder D. The neural correlates of cognitive dysfunction in phantom sounds. Brain Res. 2016 Jul; 1642:170-9.

14. Wang Y, Zhang JN, Hu W, Li JJ, Zhou JX, Zhang JP, et al. The characteristics of cognitive impairment in subjective chronic tinnitus. Brain Behav. 2018 Jan;8(3):e00918.

15. Gudwani S, Munjal SK, Panda NK, Kohli A. Association of chronic subjective tinnitus with neuro- cognitive performance. Int Tinnitus J. 2017 Dec;21(2):90-7.

16. Snowden JS. Mild cognitive impairment: aging to Alzheimer's disease. Brain. 2004 Jan;127(1):231-3.

17. Petersen RC. Mild cognitive impairment as a diagnostic entity. J Intern Med. 2004 Sep;256(3):183-94.

18. Pittman AL, Stelmachowicz PG. Hearing loss in children and adults: audiometric configuration, asymmetry, and progression. Ear Hear. 2003 Jun;24(3):198-205.

19. Lee JY, Lee DW, Cho SJ, Na DL, Jeon HJ, Kim SK, et al. Brief screening for mild cognitive impairment in elderly outpatient clinic: validation of the Korean version of the Montreal Cognitive Assessment. J Geriatr Psychiatry Neurol. 2008 Jun;21(2):104-10.

20. Kroenke K, Spitzer RL. The PHQ-9: a new depression diagnostic and severity measure. Psychiatr Ann. 2002;32(9):509-15.

21. An JY, Seo ER, Lim KH, Shin JH, Kim JB. Standardization of the Korean version of screening tool for depression (Patient Health Questionnaire-9, PHQ-9). J Korean Soc Biol Ther Psychiatry. 2013 Jun;19(1):47-56.

22. Hwang $\mathrm{IH}, \mathrm{Oh} \mathrm{DH}$. Questionnaires for Assessing Stress and Mental Health. Hanyang Med Rev. 2014 May;34(2):91-5.

23. Kang SJ, Choi SH, Lee BH, Kwon JC, Na DL, Han SH, et al. The reliability and validity of the Korean Instrumental Activities of Daily Living (K-IADL). J Korean Neurol Assoc. 2002 Jan;20(1):8-14.

24. Petersen RC. Clinical practice: mild cognitive impairment. N Engl J Med. 2011 Jun;364(23):2227-34.

25. Trevis KJ, McLachlan NM,Wilson SJ.A systematic review and metaanalysis of psychological functioning in chronic tinnitus. Clin Psychol Rev. 2018 Mar;60:62-86.

26. Oh E, Lee AY. Mild cognitive impairment. J Korean Neurol Assoc. 2016 Aug;34(3):167-75.

27. Flicker C, Ferris SH, Reisberg B. Mild cognitive impairment in the elderly: predictors of dementia. Neurology. 1991 Jul;41(7):1006-9.

28. Dag E, Bayar Muluk N, Karabicak H, Kursat Arikan O, TurkelY. Cognitive evaluation and quality of life assessment in patients with subjective tinnitus. Acta Neurol Taiwan. 2016 Mar;25(1):1-9.

29. Andersson G. Psychological aspects of tinnitus and the application of cognitive-behavioral therapy. Clin Psychol Rev. 2002 Sep;22(7): 977-90.

30. Lin FR, Metter EJ, O’Brien RJ, Resnick SM, Zonderman AB, Ferrucci L. Hearing loss and incident dementia. Arch Neurol. 2011 Feb;68(2): 214-20.

31. Vanneste S, De Ridder D. Deafferentation-based pathophysiological differences in phantom sound: tinnitus with and without hearing loss. Neuroimage. 2016 Apr;129:80-94.

32. Georgiewa P, Klapp BF, Fischer F, Reisshauer A, Juckel G, Frommer J, et al. An integrative model of developing tinnitus based on recent neurobiological findings. Med Hypotheses. 2006;66(3):592-600. 